\title{
Culturally and Economically Diverse Group
}

National Cancer Institute

\section{Source}

National Cancer Institute. Culturally and Economically Diverse Group. NCI Thesaurus.

Code C18725.

Groups of individuals that exhibit differing characteristic behavior in customs, attitudes, practices, and behaviors, or are predisposed to certain health histories due to differences in ethnicity, race, religion, national origin, or economic standing (DCCPS) 